\title{
Periodic and Chaotic Orbits of a Discrete Rational System
}

\author{
N. Lazaryan and H. Sedaghat \\ Department of Mathematics, Virginia Commonwealth University, Richmond, VA 23284-2014, USA \\ Correspondence should be addressed to H. Sedaghat; hsedagha@vcu.edu
}

Received 29 August 2014; Revised 9 January 2015; Accepted 15 January 2015

Academic Editor: Cengiz Çinar

Copyright (C) 2015 N. Lazaryan and H. Sedaghat. This is an open access article distributed under the Creative Commons Attribution License, which permits unrestricted use, distribution, and reproduction in any medium, provided the original work is properly cited.

\begin{abstract}
We study a rational planar system consisting of one linear-affine and one linear-fractional difference equation. If all of the system's parameters are positive (so that the positive quadrant is invariant and the system is continuous), then we show that the unique fixed point of the system in the positive quadrant cannot be repelling and the system does not have a snap-back repeller. By folding the system into a second-order equation, we find special cases of the system with some negative parameter values that do exhibit chaos in the sense of Li and Yorke within the positive quadrant of the plane.
\end{abstract}

\section{Introduction}

In this paper we examine the possible occurrence of coexisting cycles and Li-Yorke type chaos (see [1-5]) for the discrete planar system:

$$
\begin{gathered}
x_{n+1}=a x_{n}+b y_{n}+c, \\
y_{n+1}=\frac{a^{\prime} x_{n}+b^{\prime} y_{n}+c^{\prime}}{a^{\prime \prime} x_{n}+b^{\prime \prime} y_{n}+c^{\prime \prime}},
\end{gathered}
$$

where all 9 parameters are real numbers.

Rational equations and systems appear with increasing frequency in applications; see [6-10] for some instances of rational systems and equations in biological modeling. Specifically, a system of types (1a) and (1b) models certain stage-structured populations in biology; see [11-14]. If $A_{n}$ and $J_{n}$ denote the populations of adults and juveniles in period $n$, respectively, then the following special case of (1a) and (lb):

$$
\begin{gathered}
A_{n+1}=s_{1} J_{n}+s_{2} A_{n}, \\
J_{n+1}=\frac{b A_{n}}{1+c_{1} J_{n}+c_{2} A_{n}},
\end{gathered}
$$

is known as a stage-structured, Beverton-Holt type population model with survival rates $s_{1}, s_{2} \in(0,1)$ and fertility and competition coefficients $b, c_{1}>0, c_{2} \geq 0$. Many more discrete planar and higher dimensional models in biology, economics, and other areas involve other types of rational and nonlinear difference equations; see [15-17].

The occurrence of chaotic orbits for (1a) and (1b) is far from obvious. It is well known that a system of linear difference equations with constant coefficients does not have chaotic orbits. On the other hand, if one of the equations of the system is a polynomial of degree greater than 1 , then the system may possess chaotic orbits within a bounded invariant set, as in the case of the familiar logistic map on the real line or the Hénon map in the plane; see, for example, $[15,18]$.

We study the occurrence of complex behavior in (1a) and (1b). Prior studies of linear-fractional equations and systems (see [19] and references therein) have not been focused on demonstrating the occurrence of chaos or coexisting cycles and recent works $[20,21]$ that investigate homogeneous rational systems did not consider chaotic behavior. Studies of chaos in rational or planar systems generally do exist in the literature as indicated in the references below; see, for example, [22-26]. In particular, in [26] the occurrence of chaos in homogeneous rational systems in the plane is established.

Since (1b) is discontinuous on the plane (unlike polynomial equations), the existence of solutions is guaranteed for (1a) and (1b) only if division by zero is avoided at every step of the iteration. In typical studies of rational systems, it is generally assumed that all nine parameters and the initial values are nonnegative (we refer to this as the positive case) to avoid possible occurrence of singularities in the positive quadrant $(0, \infty)^{2}=(0, \infty) \times(0, \infty)$ of the plane. This 
quadrant is also the part of the plane that is naturally of greatest interest in modeling applications such as the aforementioned adult-juvenile model. But the type of nonlinearity exhibited by linear-fractional equations is of a particular kind that tends to be mild in nature away from singularities. This may be one reason for the relatively well-behaved orbits in the positive case rather than complex orbits that tend to be associated with rapid rates of change.

To be more precise, we show that in the positive case any fixed point $(\bar{x}, \bar{y})$ of (la) and (lb) in the positive quadrant $(\bar{x}, \bar{y}>0)$ must be nonrepelling; that is, it is not true that both of the eigenvalues of the system's linearization at $(\bar{x}, \bar{y})$ have modulus greater than 1 . This implies that $(\bar{x}, \bar{y})$ is not a snap-back repeller in the positive case.

We consider cases where some of the 9 system parameters are negative and allow singularities to occur in the positive quadrant $(0, \infty)^{2}$. For instance, if $a^{\prime \prime} b^{\prime \prime}<0$, then the straight line $a^{\prime \prime} x+b^{\prime \prime} y+c^{\prime \prime}=0$, which is part of the singularity or forbidden set of the system in this case, crosses the positive quadrant so if any point $\left(x_{n}, y_{n}\right)$ of an orbit of (1a) and (1b) falls on this line, then division by zero occurs. With negative parameters, it is necessary to either determine the forbidden sets or find a way of avoiding them. Determination of forbidden sets has been done for some higher order equations; see, for example, [27-29]. But this is a difficult task for systems like (1a) and (1b). To identify special cases of (1a) and (1b) where orbits avoid such singularities we fold the system, that is, transform it into a second-order quadratic-fractional equation and then find special cases in which the occurrence of Li-Yorke type chaos can be established in the positive quadrant. As a bonus, we find special cases of (1a) and (1b) that have periodic solutions of all possible periods in the positive quadrant. Obtaining these results would be quite difficult without folding.

Folding is applicable generically to systems, whether continuous or discrete, and used, though not by this name, in diverse areas from control theory to the study of chaos in differential systems; see [26] for an introduction in the planar case.

\section{Folding and Fixed Points}

In this section, we discuss the folding of (1a) and (1b) and its fixed points in the positive quadrant $(0, \infty)^{2}$. To avoid reductions to linear or to triangular systems, we assume throughout this paper that the parameters of (1a) and (1b) satisfy

$$
b \neq 0, \quad\left|a^{\prime}\right|+\left|a^{\prime \prime}\right|,\left|a^{\prime \prime}\right|+\left|b^{\prime \prime}\right|,\left|a^{\prime}\right|+\left|b^{\prime}\right|+\left|c^{\prime}\right|>0 .
$$

2.1. Folding the System. To fold (1a) and (1b), we solve (1a) for $y_{n}$ to obtain

$$
y_{n}=\frac{1}{b}\left(x_{n+1}-a x_{n}-c\right)
$$

Now

$$
\begin{aligned}
x_{n+2} & =a x_{n+1}+b y_{n+1}+c \\
& =c+a x_{n+1}+\frac{b a^{\prime} x_{n}+b b^{\prime} y_{n}+b c^{\prime}}{a^{\prime \prime} x_{n}+b^{\prime \prime} y_{n}+c^{\prime \prime}} .
\end{aligned}
$$

Using (1b) and (4) to eliminate $y_{n}$ yields

$$
\begin{aligned}
x_{n+2}= & c+a x_{n+1} \\
& +\frac{b a^{\prime} x_{n}+b^{\prime}\left(x_{n+1}-a x_{n}-c\right)+b c^{\prime}}{a^{\prime \prime} x_{n}+\left(b^{\prime \prime} / b\right)\left(x_{n+1}-a x_{n}-c\right)+c^{\prime \prime}} .
\end{aligned}
$$

Through combining terms and simplifying, we obtain the rational, second-order equation:

$$
x_{n+2}=a x_{n+1}+\frac{\sigma_{1} x_{n+1}+\sigma_{2} x_{n}+\sigma_{3}}{b^{\prime \prime} x_{n+1}+D_{a b}^{\prime \prime} x_{n}+D_{c b}^{\prime \prime}},
$$

where

$$
\begin{gathered}
D_{a b}^{\prime}=a^{\prime} b-a b^{\prime}, \quad D_{a b}^{\prime \prime}=a^{\prime \prime} b-a b^{\prime \prime}, \\
D_{c b}^{\prime}=b c^{\prime}-b^{\prime} c, \quad D_{c b}^{\prime \prime}=b c^{\prime \prime}-b^{\prime \prime} c, \\
\sigma_{1}=b b^{\prime}+c b^{\prime \prime}, \quad \sigma_{2}=b D_{a b}^{\prime}+c D_{a b}^{\prime \prime}, \\
\sigma_{3}=b D_{c b}^{\prime}+c D_{c b}^{\prime \prime} .
\end{gathered}
$$

We refer to the pair of (4) and (7) as a folding of (1a) and (1b). Note that (4) is a passive equation in the sense that it yields $y_{n}$ without further iterations once a solution $\left\{x_{n}\right\}$ of (7) is known. In this sense, we may think of (7) as a reduction of (1a) and (1b) to a scalar difference equation.

Equation (7) is a quadratic-fractional equation. This class of difference equations extend the linear-fractional equation and have been subjects of increasing study; see, for example, [27, 29-31].

Remark 1. A routine calculation shows that the orbits of (1a) and (1b) correspond to the solutions of (7) in the sense that if $\left\{x_{n}\right\}$ is a solution of (7) with given initial values $x_{0}$ and $x_{1}$ and $\left\{y_{n}\right\}$ is given by (4) for $n \geq 0$, then $\left\{\left(x_{n}, y_{n}\right)\right\}$ is an orbit of (1a) and (1b). Conversely, if $\left\{\left(x_{n}, y_{n}\right)\right\}$ is an orbit of (1a) and (1b) from an initial point $\left(x_{0}, y_{0}\right)$ and $x_{1}=a x_{0}+b y_{0}+c$, then $\left\{x_{n}\right\}$ is a solution of (7).

2.2. Fixed Points in the Positive Quadrant. The fixed points of (la) and (lb) satisfy the following equations:

$$
\begin{gathered}
\bar{x}=a \bar{x}+b \bar{y}+c, \\
\bar{y}=\frac{a^{\prime} \bar{x}+b^{\prime} \bar{y}+c^{\prime}}{a^{\prime \prime} \bar{x}+b^{\prime \prime} \bar{y}+c^{\prime \prime}} .
\end{gathered}
$$

From (9a),

$$
\bar{y}=\frac{(1-a) \bar{x}-c}{b} .
$$

Before calculating the values of the $x$ - and $y$-components, we note the following facts about the solutions of the system (1a) and (1b). 
Lemma 2. Assume that all system parameters are nonnegative and satisfy (3); that is,

$$
b>0, \quad a^{\prime}+a^{\prime \prime}, a^{\prime \prime}+b^{\prime \prime}, a^{\prime}+b^{\prime}+c^{\prime}>0 .
$$

(a) If there is a fixed point $(\bar{x}, \bar{y})$ of the system in the positive quadrant (i.e., $\bar{x}, \bar{y}>0$ ), then $0 \leq a<1$ and $\bar{x}>$ $c /(1-a)$.

(b) If $a>1$, then every orbit of (1a) and (1b) in the positive quadrant is unbounded.

Proof. (a) Let $(\bar{x}, \bar{y})$ be a fixed point of the system in the positive quadrant. Then, by $(9 \mathrm{a})$

$$
(1-a) \bar{x}=b \bar{y}+c>c \geq 0
$$

since $b, \bar{y}>0$ by hypothesis and (11). Since $\bar{x}>0$, it follows that $1-a>0$ or $a<1$.

(b) From (1a), it follows that for all $n$

$$
x_{n+1}=a x_{n}+b y_{n}+c \geq a x_{n} .
$$

By induction, $x_{n} \geq a^{n} x_{0}$ for all $n$ and it follows that the orbit is unbounded if $x_{0}>0$.

Now, to calculate the fixed points, from (10) and (9b), we obtain

$$
\begin{aligned}
\frac{(1-a) \bar{x}-c}{b} & =\frac{a^{\prime} \bar{x}+b^{\prime}[(1-a) \bar{x}-c] / b+c^{\prime}}{a^{\prime \prime} \bar{x}+b^{\prime \prime}[(1-a) \bar{x}-c] / b+c^{\prime \prime}} \\
& =\frac{a^{\prime} b \bar{x}+b^{\prime}(1-a) \bar{x}-b^{\prime} c+b c^{\prime}}{a^{\prime \prime} b \bar{x}+b^{\prime \prime}(1-a) \bar{x}-b^{\prime \prime} c+b c^{\prime \prime}} \\
& =\frac{\left(D_{a b}^{\prime}+b^{\prime}\right) \bar{x}+D_{c b}^{\prime}}{\left(D_{a b}^{\prime \prime}+b^{\prime \prime}\right) \bar{x}+D_{c b}^{\prime \prime}} .
\end{aligned}
$$

$$
\begin{aligned}
\bar{x}= & \frac{d_{2} \pm \sqrt{d_{2}^{2}+4 d_{1} d_{3}}}{2 d_{1}} \\
= & \frac{b\left(D_{a b}^{\prime \prime}+b^{\prime}\right)+c\left(D_{a b}^{\prime \prime}+b^{\prime \prime}\right)-(1-a) D_{c b}^{\prime \prime}}{2(1-a)\left(D_{a b}^{\prime \prime}+b^{\prime \prime}\right)} \\
& \pm \frac{\sqrt{\left[b\left(D_{a b}^{\prime \prime}+b^{\prime}\right)+c\left(D_{a b}^{\prime \prime}+b^{\prime \prime}\right)-(1-a) D_{c b}^{\prime \prime}\right]^{2}+4(1-a)\left(D_{a b}^{\prime \prime}+b^{\prime \prime}\right)\left(c D_{c b}^{\prime \prime}+b D_{c b}^{\prime}\right)}}{2(1-a)\left(D_{a b}^{\prime \prime}+b^{\prime \prime}\right)} .
\end{aligned}
$$

These roots can be expressed more succinctly using the parameters of the folding. We use the notation $\bar{x}$ for the root with the positive sign:

$$
\bar{x}=\frac{\sigma_{1}+\sigma_{2}-(1-a) D_{c b}^{\prime \prime}+\sqrt{\left[\sigma_{1}+\sigma_{2}-(1-a) D_{c b}^{\prime \prime}\right]^{2}+4(1-a)\left(b^{\prime \prime}+D_{a b}^{\prime \prime}\right) \sigma_{3}}}{2(1-a)\left(b^{\prime \prime}+D_{a b}^{\prime \prime}\right)}
$$


with $\bar{y}$ given by (10) and use $\tilde{x}$ to denote the root with the negative sign:

$$
\tilde{x}=\frac{\sigma_{1}+\sigma_{2}-(1-a) D_{c b}^{\prime \prime}-\sqrt{\left[\sigma_{1}+\sigma_{2}-(1-a) D_{c b}^{\prime \prime}\right]^{2}+4(1-a)\left(b^{\prime \prime}+D_{a b}^{\prime \prime}\right) \sigma_{3}}}{2(1-a)\left(b^{\prime \prime}+D_{a b}^{\prime \prime}\right)}
$$

with $\tilde{y}$ again given by (10). It is an interesting fact that of the two fixed points above only one of them can be in the positive quadrant.

Lemma 3. Let all system parameters in (1a) and (1b) be nonnegative and satisfy (11). If (1a) and (1b) have a fixed point in $(0, \infty)^{2}$, then that fixed point is $(\bar{x}, \bar{y})$ and it is unique with $\bar{x}$ given by (20) and $\bar{y}$ given by (10).

Proof. Lemma 2 and the above discussion indicate that a necessary condition for the existence of fixed points in the positive quadrant is that $0 \leq a<1$ holds. We found two possible fixed points given by (20) and (21) plus (10). Both of these are well defined if and only if (18) holds. Now, again by Lemma 2 , the fixed point $(\bar{x}, \bar{y})$ is in the positive quadrant if $\bar{x}>c /(1-a)$; that is,

$$
\begin{gathered}
d_{2}+\sqrt{d_{2}^{2}+4 d_{1} d_{3}}>\frac{2 c d_{1}}{1-a} \\
\sqrt{d_{2}^{2}+4(1-a)\left(b^{\prime \prime}+D_{a b}^{\prime \prime}\right) \sigma_{3}}>2 c\left(b^{\prime \prime}+D_{a b}^{\prime \prime}\right)-d_{2} \\
(1-a) \sigma_{3}>c^{2}\left(b^{\prime \prime}+D_{a b}^{\prime \prime}\right)-c d_{2}
\end{gathered}
$$

Similarly for $\tilde{x}$, it is required that

$$
\begin{gathered}
d_{2}-\sqrt{d_{2}^{2}+4 d_{1} d_{3}}>\frac{2 c d_{1}}{1-a} \\
\sqrt{d_{2}^{2}+4(1-a)\left(b^{\prime \prime}+D_{a b}^{\prime \prime}\right) \sigma_{3}}<d_{2}-2 c\left(b^{\prime \prime}+D_{a b}^{\prime \prime}\right) \\
(1-a) \sigma_{3}<c^{2}\left(b^{\prime \prime}+D_{a b}^{\prime \prime}\right)-c d_{2} .
\end{gathered}
$$

The preceding covers all possible fixed points in the first quadrant under the hypotheses of the lemma. We now show that (23) cannot hold, thus leaving $(\bar{x}, \bar{y})$ as the only possible fixed point in the first quadrant. Note that

$$
\begin{aligned}
c d_{2}- & c^{2}\left(b^{\prime \prime}+D_{a b}^{\prime \prime}\right) \\
= & c\left[\sigma_{1}+\sigma_{2}-(1-a) D_{c b}^{\prime \prime}\right]-c^{2} b^{\prime \prime}-c^{2} D_{a b}^{\prime \prime} \\
= & c\left[b b^{\prime}+b^{\prime \prime} c+b\left(a^{\prime} b-a b^{\prime}\right)+c D_{a b}^{\prime \prime}-(1-a) D_{c b}^{\prime \prime}\right] \\
& -c^{2} b^{\prime \prime}-c^{2} D_{a b}^{\prime \prime} \\
= & (1-a) b b^{\prime} c+a^{\prime} b^{2} c-c(1-a) D_{c b}^{\prime \prime} .
\end{aligned}
$$

\section{Therefore,}

$$
\begin{aligned}
(1 & -a) \sigma_{3}+c d_{2}-c^{2}\left(b^{\prime \prime}+D_{a b}^{\prime \prime}\right) \\
& =(1-a) b D_{c b}^{\prime}+(1-a) b b^{\prime} c+a^{\prime} b^{2} c \\
& =(1-a) b\left(c^{\prime} b-b^{\prime} c\right)+(1-a) b b^{\prime} c+a^{\prime} b^{2} c \\
& =(1-a) b^{2} c^{\prime}+a^{\prime} b^{2} c .
\end{aligned}
$$

Since the last quantity is nonnegative under the hypotheses, it follows that (23) does not hold and the proof is complete.

\section{Nonexistence of Repellers}

We see in the proof of Lemma 3 that $(\bar{x}, \bar{y})$ exists in the positive quadrant if (18) and (22) both hold. Of particular interest to us is whether $(\bar{x}, \bar{y})$ can be repelling under the hypotheses of Lemma 3. We recall that a fixed point is repelling if all eigenvalues of the linearization of the system at that point have modulus greater than 1 .

Theorem 4. Let all system parameters in (1a) and (1b) be nonnegative and satisfy (11). If (1a) and (1b) have a fixed point in $(0, \infty)^{2}$, then it is uniquely $(\bar{x}, \bar{y})$ and this is not a repelling fixed point.

Proof. The first assertion follows from Lemma 3. To show that $(\bar{x}, \bar{y})$ is not repelling, we examine the eigenvalues of the linearization of (1a) and (1b) at $(\bar{x}, \bar{y})$. The Jacobian matrix of (1a) and (1b) evaluated at the fixed point $(\bar{x}, \bar{y})$ is

$$
J(\bar{x}, \bar{y})=\left(\begin{array}{ll}
a & b \\
p & q
\end{array}\right)
$$

where

$$
\begin{aligned}
& p=\frac{a^{\prime}\left(a^{\prime \prime} \bar{x}+b^{\prime \prime} \bar{y}+c^{\prime \prime}\right)-a^{\prime \prime}\left(a^{\prime} \bar{x}+b^{\prime} \bar{y}+c^{\prime}\right)}{\left(a^{\prime \prime} \bar{x}+b^{\prime \prime} \bar{y}+c^{\prime \prime}\right)^{2}}, \\
& q=\frac{b^{\prime}\left(a^{\prime \prime} \bar{x}+b^{\prime \prime} \bar{y}+c^{\prime \prime}\right)-b^{\prime \prime}\left(a^{\prime} \bar{x}+b^{\prime} \bar{y}+c^{\prime}\right)}{\left(a^{\prime \prime} \bar{x}+b^{\prime \prime} \bar{y}+c^{\prime \prime}\right)^{2}} .
\end{aligned}
$$

Since by (9b)

$$
a^{\prime} \bar{x}+b^{\prime} \bar{y}+c^{\prime}=\bar{y}\left(a^{\prime \prime} \bar{x}+b^{\prime \prime} \bar{y}+c^{\prime \prime}\right),
$$

the above expressions for $p$ and $q$ reduce to

$$
p=\frac{a^{\prime}-a^{\prime \prime} \bar{y}}{a^{\prime \prime} \bar{x}+b^{\prime \prime} \bar{y}+c^{\prime \prime}}, \quad q=\frac{b^{\prime}-b^{\prime \prime} \bar{y}}{a^{\prime \prime} \bar{x}+b^{\prime \prime} \bar{y}+c^{\prime \prime}} .
$$


The characteristic equation of the above Jacobian is

$$
\lambda^{2}-(a+q) \lambda-(b p-a q)=0,
$$

where

$$
\begin{aligned}
a+q & =a+\frac{b^{\prime}-b^{\prime \prime} \bar{y}}{a^{\prime \prime} \bar{x}+b^{\prime \prime} \bar{y}+c^{\prime \prime}} \\
& =\frac{a a^{\prime \prime} \bar{x}-(1-a) b^{\prime \prime} \bar{y}+a c^{\prime \prime}+b^{\prime}}{a^{\prime \prime} \bar{x}+b^{\prime \prime} \bar{y}+c^{\prime \prime}}, \\
b p-a q & =\frac{a^{\prime} b-a^{\prime \prime} b \bar{y}-a b^{\prime}+a b^{\prime \prime} \bar{y}}{a^{\prime \prime} \bar{x}+b^{\prime \prime} \bar{y}+c^{\prime \prime}} \\
& =\frac{D_{a b}^{\prime}-D_{a b}^{\prime \prime} \bar{y}}{a^{\prime \prime} \bar{x}+b^{\prime \prime} \bar{y}+c^{\prime \prime}} .
\end{aligned}
$$

Let

$$
\alpha=a+q, \quad \beta=b p-a q
$$

and write (30) as

$$
\lambda^{2}-\alpha \lambda-\beta=0
$$

The roots of (33) are the eigenvalues; that is,

$$
\lambda_{1}=\frac{\alpha-\sqrt{\alpha^{2}+4 \beta}}{2}, \quad \lambda_{2}=\frac{\alpha+\sqrt{\alpha^{2}+4 \beta}}{2} .
$$

When $\alpha^{2}+4 \beta<0$ (or $\beta<-\alpha^{2} / 4$ ), the eigenvalues $\lambda_{1}, \lambda_{2}$ are complex and their common modulus is $-\beta$. So both eigenvalues have modulus greater than 1 if and only if

$$
\beta<-1 \text {. }
$$

If $\alpha^{2}+4 \beta \geq 0$, then both eigenvalues are real with $\lambda_{1} \leq$ $\alpha / 2 \leq \lambda_{2}$. By considering the 3 possible cases,

$$
\begin{aligned}
& \lambda_{1}, \lambda_{2}<-1, \quad \lambda_{1}, \lambda_{2}>1 \text { or } \\
& \lambda_{1}<-1, \quad \lambda_{2}>1
\end{aligned}
$$

routine calculations show that both eigenvalues have modulus greater than 1 if and only if

$$
2<|\alpha|<1-\beta \quad \text { or } \beta>1+|\alpha| \text {. }
$$

With regard to (35), note that by (10) $\bar{x}-b \bar{y}=a \bar{x}+c$ so $\beta+1$

$$
\begin{aligned}
& =\frac{D_{a b}^{\prime}-D_{a b}^{\prime \prime} \bar{y}+a^{\prime \prime} \bar{x}+b^{\prime \prime} \bar{y}+c^{\prime \prime}}{a^{\prime \prime} \bar{x}+b^{\prime \prime} \bar{y}+c^{\prime \prime}} \\
& =\frac{a^{\prime} b-a b^{\prime}+a^{\prime \prime}(\bar{x}-b \bar{y})+a b^{\prime \prime} \bar{y}+b^{\prime \prime} \bar{y}+c^{\prime \prime}}{a^{\prime \prime} \bar{x}+b^{\prime \prime} \bar{y}+c^{\prime \prime}} \\
& =\frac{a^{\prime} b-a b^{\prime}+a^{\prime \prime} c+a\left(a^{\prime \prime} \bar{x}+b^{\prime \prime} \bar{y}\right)+b^{\prime \prime} \bar{y}+c^{\prime \prime}}{a^{\prime \prime} \bar{x}+b^{\prime \prime} \bar{y}+c^{\prime \prime}} .
\end{aligned}
$$

By (28),

$$
\begin{aligned}
a^{\prime \prime} \bar{x}+b^{\prime \prime} \bar{y} & =\frac{a^{\prime} \bar{x}+b^{\prime} \bar{y}+c^{\prime}}{\bar{y}}-c^{\prime \prime} \\
& =\frac{a^{\prime} \bar{x}+c^{\prime}}{\bar{y}}+b^{\prime}-c^{\prime \prime}
\end{aligned}
$$

so

$$
\begin{aligned}
\beta+1 & \\
= & \frac{a^{\prime} b+a^{\prime \prime} c+a\left(a^{\prime} \bar{x}+c^{\prime}\right) / \bar{y}+(1-a) c^{\prime \prime}+b^{\prime \prime} \bar{y}}{a^{\prime \prime} \bar{x}+b^{\prime \prime} \bar{y}+c^{\prime \prime}} \geq 0 .
\end{aligned}
$$

It follows that (35) does not hold and further $1-\beta \leq 2$ so that the first of the inequalities in (37) also does not hold. To check the remaining inequality $\beta>1+|\alpha|$, it is more convenient if we rewrite the expressions for $\alpha, \beta$ in terms of the folding parameters, using (10) to eliminate $\bar{y}$

$$
\begin{gathered}
\alpha=\frac{\left[(2 a-1) b^{\prime \prime}+a D_{a b}^{\prime \prime}\right] \bar{x}+\sigma_{1}+a D_{c b}^{\prime \prime}}{\left(b^{\prime \prime}+D_{a b}^{\prime \prime}\right) \bar{x}+D_{c b}^{\prime \prime}}, \\
\beta=\frac{\sigma_{2}-(1-a) D_{a b}^{\prime \prime} \bar{x}}{\left(b^{\prime \prime}+D_{a b}^{\prime \prime}\right) \bar{x}+D_{c b}^{\prime \prime}} .
\end{gathered}
$$

Note that

$$
\begin{aligned}
& \left(b^{\prime \prime}+D_{a b}^{\prime \prime}\right) \bar{x}+D_{c b}^{\prime \prime} \\
& >\frac{c\left[a^{\prime \prime} b+(1-a) b^{\prime \prime}\right]}{1-a}+b c^{\prime \prime}-b^{\prime \prime} c \\
& =\frac{a^{\prime \prime} b c+(1-a) b^{\prime \prime} c+(1-a)\left(b c^{\prime \prime}-b^{\prime \prime} c\right)}{1-a} \\
& =\frac{a^{\prime \prime} b c+(1-a) b c^{\prime \prime}}{1-a} \geq 0
\end{aligned}
$$

so $\beta>1-\alpha$ if and only if

$$
\begin{aligned}
& {\left[(2 a-1) b^{\prime \prime}+a D_{a b}^{\prime \prime}\right] \bar{x}+\sigma_{1}+a D_{c b}^{\prime \prime}} \\
& \quad>D_{c b}^{\prime \prime}+\left(b^{\prime \prime}+D_{a b}^{\prime \prime}\right) \bar{x}-\sigma_{2}+(1-a) D_{a b}^{\prime \prime} \bar{x}
\end{aligned}
$$

which reduces to

$$
2(1-a)\left(b^{\prime \prime}+D_{a b}^{\prime \prime}\right) \bar{x}<\sigma_{1}+\sigma_{2}-(1-a) D_{c b}^{\prime \prime} .
$$

However, (20) implies that

$$
2(1-a)\left(b^{\prime \prime}+D_{a b}^{\prime \prime}\right) \bar{x} \geq \sigma_{1}+\sigma_{2}-(1-a) D_{c b}^{\prime \prime} .
$$

So (44) is false, and thus $\alpha \leq 1-\beta$ or equivalently $\beta \leq$ $1-\alpha \leq 1+|\alpha|$. Hence, $(\bar{x}, \bar{y})$ is not repelling in the positive quadrant.

The above theorem shows that any fixed point of the system in the positive quadrant is nonrepelling if all system parameters are nonnegative; in particular, there are no snapback repellers in the positive case (though unstable saddle fixed points exist for some parameter values). 


\section{Cycles and Chaos in the Positive Quadrant}

If $a=0$, then (7) reduces to the linear-fractional equation:

$$
x_{n+2}=\frac{\sigma_{1} x_{n+1}+\sigma_{2} x_{n}+\sigma_{3}}{b^{\prime \prime} x_{n+1}+D_{a b}^{\prime \prime} x_{n}+D_{c b}^{\prime \prime}} .
$$

This type of linear-fractional equation has been studied extensively under the assumption of nonnegative parameters; see, for example, [19]. Although many questions remain to be answered about (46), chaotic solutions for it have not been found. To assure the occurrence of limit cycles and chaos and to avoid reductions to linear systems or to triangular systems where one of the equations is single variable, we assume that

$$
a, b, a^{\prime}, b^{\prime \prime} \neq 0 \text {. }
$$

If some of the parameters in (7) are negative, then even the existence and boundedness of solutions are nontrivial issues. Our aim here is to show that special cases of (7) with some negative coefficients exhibit Li-Yorke chaos in the positive quadrant. We note that $(7)$ reduces to a first-order difference equation if

$$
D_{a b}^{\prime}=D_{a b}^{\prime \prime}=0
$$

In this case, we define $r_{n}=x_{n+1}$ and $r_{0}=x_{1}=a x_{0}+b y_{0}+c$ to obtain

$$
r_{n+1}=a r_{n}+\frac{\sigma_{1} r_{n}+\sigma_{3}}{b^{\prime \prime} r_{n}+D_{c b}^{\prime \prime}}
$$

The theory of one-dimensional maps may be applied to (49). To simplify calculations, we assume in addition to (48) that

$$
D_{c b}^{\prime \prime}=0, \quad D_{c b}^{\prime} \neq 0
$$

which reduce $(49)$ to

$$
r_{n+1}=a r_{n}+q+\frac{s}{r_{n}},
$$

where $q=c+b b^{\prime} / b^{\prime \prime}$ and $s=b D_{c b}^{\prime} / b^{\prime \prime}$.

Note that if $D_{c b}^{\prime}=0$, then (51) is affine and as such it does not have chaotic solutions.

A comprehensive study of (51) appears in [30]. The following is a consequence of the results in [30]. We point out that if $p$ is the minimal period of a solution $\left\{r_{n}\right\}$ of (51) with $r_{0}>$ 0 , then the sequence $\left\{x_{n}\right\}$ also has minimal period $p$ and by (4) $\left\{y_{n}\right\}$ has period $p$. It follows that the orbit $\left\{\left(x_{n}, y_{n}\right)\right\}$ has minimal period $p$.

Theorem 5. Assume that conditions (47), (48), and (50) hold with the (normalized) values $a=1$ and $b^{\prime \prime}=b D_{c b}^{\prime}$ and define $q=c+b b^{\prime} / b^{\prime \prime}$.

(a) If $-2<q<0$, then all orbits of (1a) and (1b) with $x_{0}+b y_{0}+c>0$ are well defined and bounded. If also $b^{\prime} / b^{\prime \prime}>0$, then these orbits are contained in $(0, \infty)^{2}$.

(b) If $-\sqrt{2}<q<0$, then all orbits of (1a) and (1b) with $x_{0}+b y_{0}+c>0$ converge to the unique fixed point $(\bar{x}, \bar{y})=(-1 / q,-c / b)$ of $(1 a)$ and $(1 b)$. (c) If $-\sqrt{5 / 2}<q<-\sqrt{2}$, then (1a) and (1b) have an asymptotically stable 2-cycle $\left\{\left(x_{1}, y_{1}\right),\left(x_{2}, y_{2}\right)\right\}$ where $y_{i}$ is given by (4) and

$$
x_{1}=\frac{-q-\sqrt{q^{2}-2}}{2}, \quad x_{2}=\frac{-q+\sqrt{q^{2}-2}}{2} .
$$

(d) If $q=-\sqrt{3}$ and $x_{0}+b y_{0}+c=2(1+\cos \pi / 9) / \sqrt{3}$, then the points $\left(x_{i}, y_{i}\right), i=1,2,3$, constitute a stable orbit of period 3 for (1a) and (1b) where $y_{i}$ is given by (4) and

$$
\begin{gathered}
x_{1}=\frac{2}{\sqrt{3}}\left(1+\cos \frac{\pi}{9}\right), \quad x_{2}=x_{1}-\sqrt{3}+\frac{1}{x_{1}}, \\
x_{3}=x_{2}-\sqrt{3}+\frac{1}{x_{2}} .
\end{gathered}
$$

(e) If $-2<q \leq-\sqrt{3}$, then orbits of (1a) and (1b) with $x_{0}+b y_{0}+c>0$ include cycles of all possible periods.

(f) For $-2<q<-\sqrt{3}$, orbits of (1a) and (1b) with $x_{0}+$ $b y_{0}+c>0$ are bounded and exhibit chaotic behavior.

Proof. Statements (a)-(f) follow largely from Theorems 4-6 in [30]. It only remains to show that orbits whose initial points satisfy $x_{0}+b y_{0}+c>0$ are contained in $(0, \infty)^{2}$ and to determine the unique fixed point. Since $x_{n}=r_{n-1}>0$ for all $n \geq 1$, (4) and (51) imply under the assumptions in (a) that

$$
\begin{aligned}
y_{n} & =\frac{1}{b}\left(r_{n}-a r_{n-1}-c\right) \\
& =-\frac{c}{b}+\frac{1}{b}\left(q+\frac{1}{r_{n-1}}\right) \\
& =-\frac{c}{b}+\frac{c}{b}+\frac{b^{\prime}}{b^{\prime \prime}}+\frac{1}{b r_{n-1}} \\
& =\frac{b^{\prime}}{b^{\prime \prime}}\left(1+\frac{1}{r_{n-1}}\right) .
\end{aligned}
$$

If $b^{\prime} / b^{\prime \prime}>0$, then it follows that $y_{n}>0$ for all $n \geq 1$ and the proof of (a) is complete. Finally, in (b) we see that the fixed point of (1a) and (1b) when $a=1$ is determined from (9a) and (9b), (48), and (50) as

$$
\left(\frac{-b^{\prime \prime}}{b b^{\prime}+b^{\prime \prime} c},-\frac{c}{b}\right)=\left(-\frac{1}{q},-\frac{c}{b}\right)
$$

which is in the positive quadrant if $q, c / b<0$.

Example 6. To illustrate Theorem 5, consider the following special case of (1a) and (1b):

$$
\begin{gathered}
x_{n+1}=x_{n}+2 y_{n}-2, \\
y_{n+1}=\frac{0.75 x_{n}+1.5 y_{n}}{3 x_{n}+6 y_{n}-6}
\end{gathered}
$$

which satisfies Part (c) of Theorem $5(q=-1.5)$ and there exists an asymptotically stable 2 -cycle $\{(1,0.75),(0.5,1.25)\}$ 
(a limit cycle) for this system. Different parameter values yield the following system which satisfies Parts (e) and (f) of Theorem 5 with $q \approx-1.83$ :

$$
\begin{gathered}
x_{n+1}=x_{n}+2 y_{n}-2, \\
y_{n+1}=\frac{0.25 x_{n}+0.5 y_{n}+1}{3 x_{n}+6 y_{n}-6} .
\end{gathered}
$$

This special case of (1a) and (1b) has periodic orbits of all periods (depending on initial points) and exhibits LiYorke type chaos. This fact is far from obvious and even the existence of cycles in the first quadrant for these equations is quite difficult to prove without folding.

We also mention that $b^{\prime} / b^{\prime \prime}>0$ in both of the above systems so every orbit whose initial point $\left(x_{0}, y_{0}\right)$ satisfies $x_{0}+b y_{0}+c>0$ is contained in the positive quadrant $(0, \infty)^{2}$.

The hypotheses of Theorem 5 are sufficient but not necessary for the occurrence of complex behavior in the positive quadrant. In fact, due to the continuity of rational expressions in terms of their parameters, the conclusions of Theorem 5 hold if the quantities $D_{a b}^{\prime}, D_{a b}^{\prime \prime}$, and $D_{c b}^{\prime \prime}$ are sufficiently small but not necessarily zero. Numerical simulations indicate that Li-Yorke chaos persists in the positive quadrant if the parameters in the last system above are slightly perturbed. Caution is needed though because if we deviate too much from the conditions of Theorem 5 , then the nontrivial nature of the singularity set must be taken into account before a claim of the occurrence of chaos can be verified.

\section{Conflict of Interests}

The authors declare that there is no conflict of interests regarding the publication of this paper.

\section{Acknowledgment}

The authors appreciate beneficial comments by an anonymous referee.

\section{References}

[1] T. Y. Li and J. A. Yorke, "Period three implies chaos," The American Mathematical Monthly, vol. 82, no. 10, pp. 985-992, 1975.

[2] F. R. Marotto, "Snap-back repellers imply chaos in $\mathbb{R}^{n}$ ", Journal of Mathematical Analysis and Applications, vol. 63, no. 1, pp. 199223, 1978.

[3] F. R. Marotto, "On redefining a snap-back repeller," Chaos, Solitons and Fractals, vol. 25, no. 1, pp. 25-28, 2005.

[4] Y. Shi and P. Yu, "Chaos induced by regular snap-back repellers," Journal of Mathematical Analysis and Applications, vol. 337, no. 2, pp. 1480-1494, 2008.

[5] C. Tian and G. Chen, "Chaos in the sense of Li-Yorke in coupled map lattices," Physica A, vol. 376, pp. 246-252, 2007.

[6] Z. AlSharawi and M. Rhouma, "Coexistence and extinction in a competitive exclusion Leslie/Gower model with harvesting and stocking," Journal of Difference Equations and Applications, vol. 15, no. 11-12, pp. 1031-1053, 2009.
[7] A. J. Harry, C. M. Kent, and V. L. Kocic, "Global behavior of solutions of a periodically forced Sigmoid Beverton-Holt model," Journal of Biological Dynamics, vol. 6, no. 2, pp. 212234, 2012.

[8] S. Kalabušić, M. R. S. Kulenović, and E. Pilav, "Dynamics of a two-dimensional system of rational difference equations of Leslie-Gower type," Advances in Difference Equations, vol. 2011, article 29, 2011.

[9] Y. Kang and H. Smith, "Global dynamics of a discrete twospecies lottery-Ricker competition model," Journal of Biological Dynamics, vol. 6, no. 2, pp. 358-376, 2012.

[10] E. C. Pielou, Population and Community Ecology, Gordon and Breach, New York, NY, USA, 1974.

[11] A. S. Ackleh and S. R. Jang, "A discrete two-stage population model: continuous versus seasonal reproduction," Journal of Difference Equations and Applications, vol. 13, no. 4, pp. 261-274, 2007.

[12] J. M. Cushing, "A juvenile-adult model with periodic vital rates," Journal of Mathematical Biology, vol. 53, no. 4, pp. 520-539, 2006.

[13] G. Giordano and F. Lutscher, "Harvesting and predation of a sex- and age-structured population," Journal of Biological Dynamics, vol. 5, no. 6, pp. 600-618, 2011.

[14] E. Liz and P. Pilarczyk, "Global dynamics in a stage-structured discrete-time population model with harvesting," Journal of Theoretical Biology, vol. 297, pp. 148-165, 2012.

[15] S. Elaydi, An Introduction to Difference Equations, Springer, New York, NY, USA, 3rd edition, 2005.

[16] V. L. Kocic and G. Ladas, Global Behavior of Nonlinear Difference Equations of Higher Order with Applications, vol. 256 of Mathematics and its Applications, Kluwer Academic, Dordrecht, The Netherlands, 1993.

[17] H. Sedaghat, Nonlinear Difference Equations: Theory with Applications to Social Science Models, Kluwer Academic, Dordrecht, The Netherlands, 2003.

[18] P. G. Drazin, Nonlinear Systems, Cambridge Texts in Applied Mathematics, Cambridge University Press, Cambridge, UK, 1992.

[19] M. R. Kulenovic and G. Ladas, Dynamics of Second Order Rational Difference Equations, Chapman \& Hall/CRC, Boca Raton, FL, Boca Raton, Fla, USA, 2002.

[20] Y. S. Huang and P. M. Knopf, "Global convergence properties of first-order homogeneous systems of rational difference equations," Journal of Difference Equations and Applications, vol. 18, no. 10, pp. 1683-1707, 2012.

[21] R. Mazrooei-Sebdani, "Homogeneous rational systems of difference equations in the plane," Journal of Difference Equations and Applications, vol. 19, no. 2, pp. 273-303, 2013.

[22] G.-I. Bischi, L. Gardini, and C. Mira, "Maps with a vanishing denominator. A survey of some results," Nonlinear Analysis: Theory, Methods \& Applications, vol. 47, no. 4, pp. 2171-2185, 2001.

[23] G.-I. Bischi, L. Gardini, and C. Mira, "Plane maps with denominator. Part II. Noninvertible maps with simple focal points," International Journal of Bifurcation and Chaos, vol. 13, no. 8, pp. 2253-2277, 2003.

[24] G.-I. Bischi, L. Gardini, and C. Mira, "Plane maps with denominator. I. Some generic properties," International Journal of Bifurcation and Chaos in Applied Sciences and Engineering, vol. 9, no. 1, pp. 119-153, 1999. 
[25] G. I. Bischi, L. Gardini, and D. Fournier-Prunaret, "Plane maps with denominator. Part III: nonsimple focal points and related bifurcations," International Journal of Bifurcation and Chaos, vol. 15, no. 2, pp. 451-496, 2005.

[26] H. Sedaghat, "Folding, cycles and chaos in planar systems," Journal of Difference Equations and Applications, vol. 21, no. 1, pp. 1-15, 2015.

[27] M. Dehghan, R. Mazrooei-Sebdani, and H. Sedaghat, "Global behaviour of the Riccati difference equation of order two," Journal of Difference Equations and Applications, vol. 17, no. 4, pp. 467-477, 2011.

[28] F. J. Palladino, "On invariants and forbidden sets," http://arxivweb3.library.cornell.edu/abs/1203.2170v2.

[29] H. Sedaghat, "Global behaviours of rational difference equations of orders two and three with quadratic terms," Journal of Difference Equations and Applications, vol. 15, no. 3, pp. 215-224, 2009.

[30] M. Dehghan, C. M. Kent, R. Mazrooei-Sebdani, N. L. Ortiz, and H. Sedaghat, "Dynamics of rational difference equations containing quadratic terms," Journal of Difference Equations and Applications, vol. 14, no. 2, pp. 191-208, 2008.

[31] S. Kalabušić, M. R. S. Kulenović, and M. Mehuljić, "Global period-doubling bifurcation of quadratic fractional second order difference equation," Discrete Dynamics in Nature and Society, vol. 2014, Article ID 920410, 13 pages, 2014. 


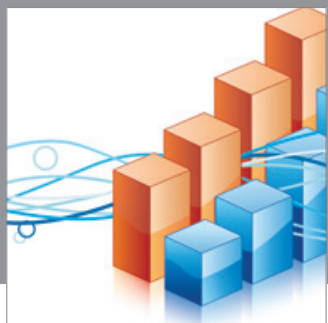

Advances in

Operations Research

mansans

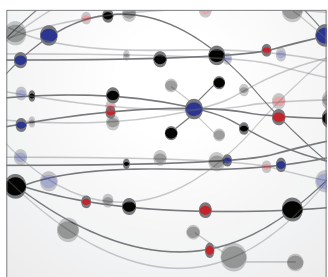

The Scientific World Journal
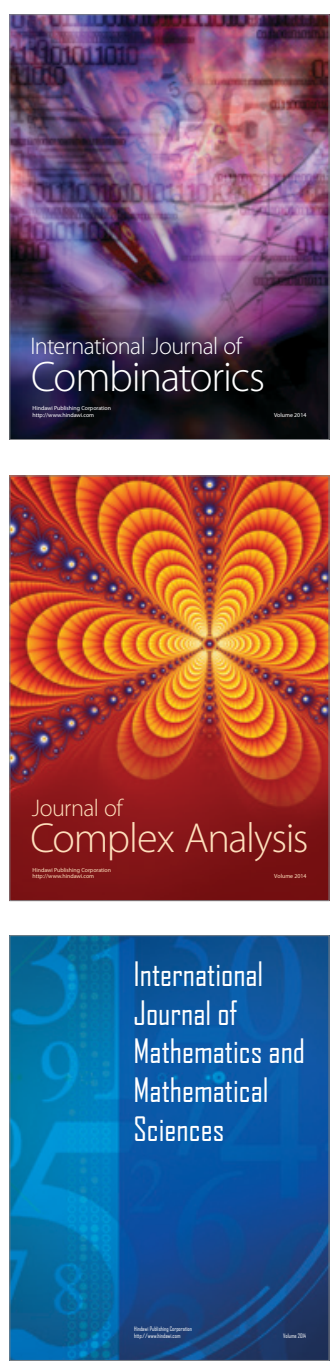
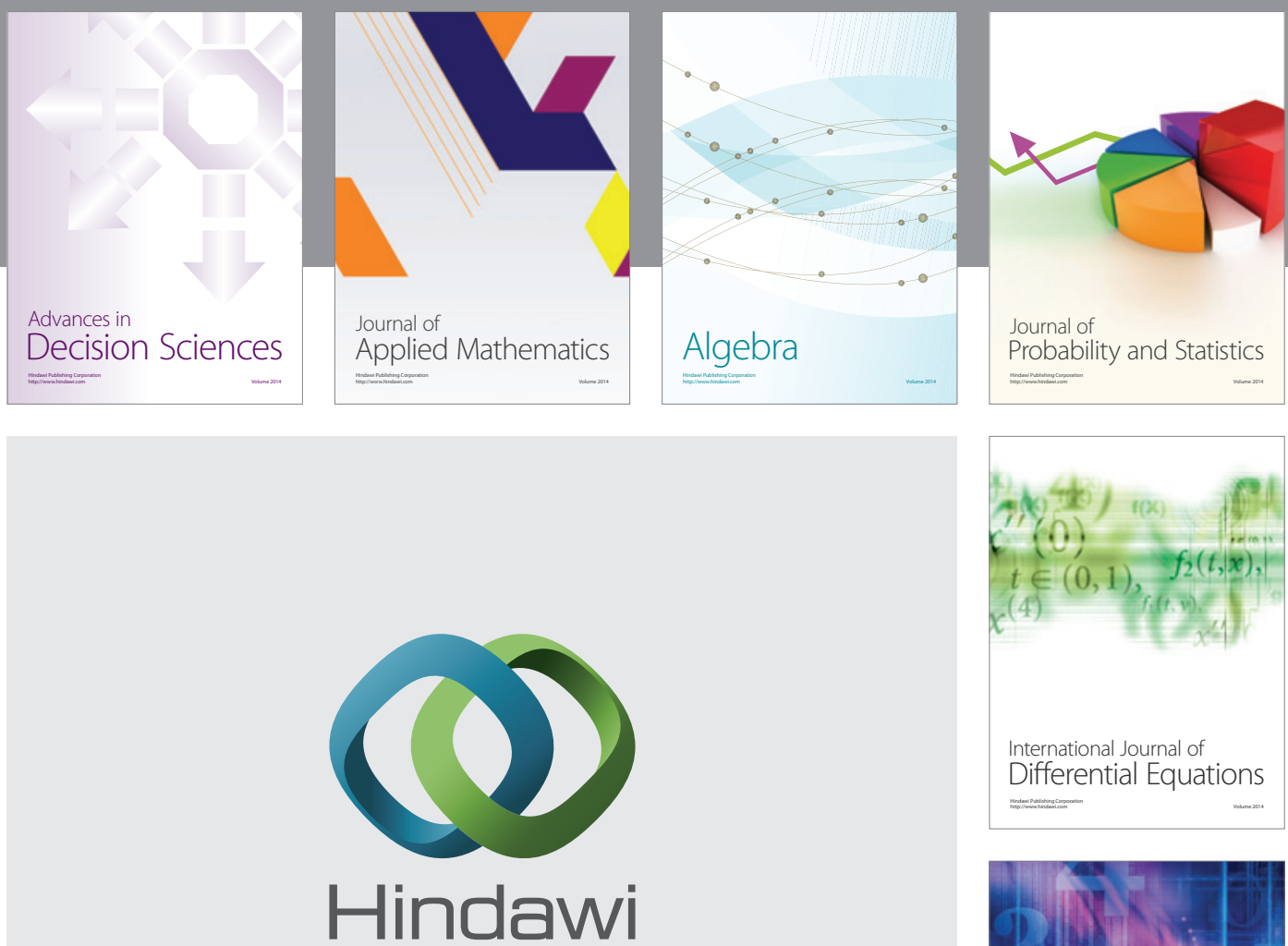

Submit your manuscripts at http://www.hindawi.com
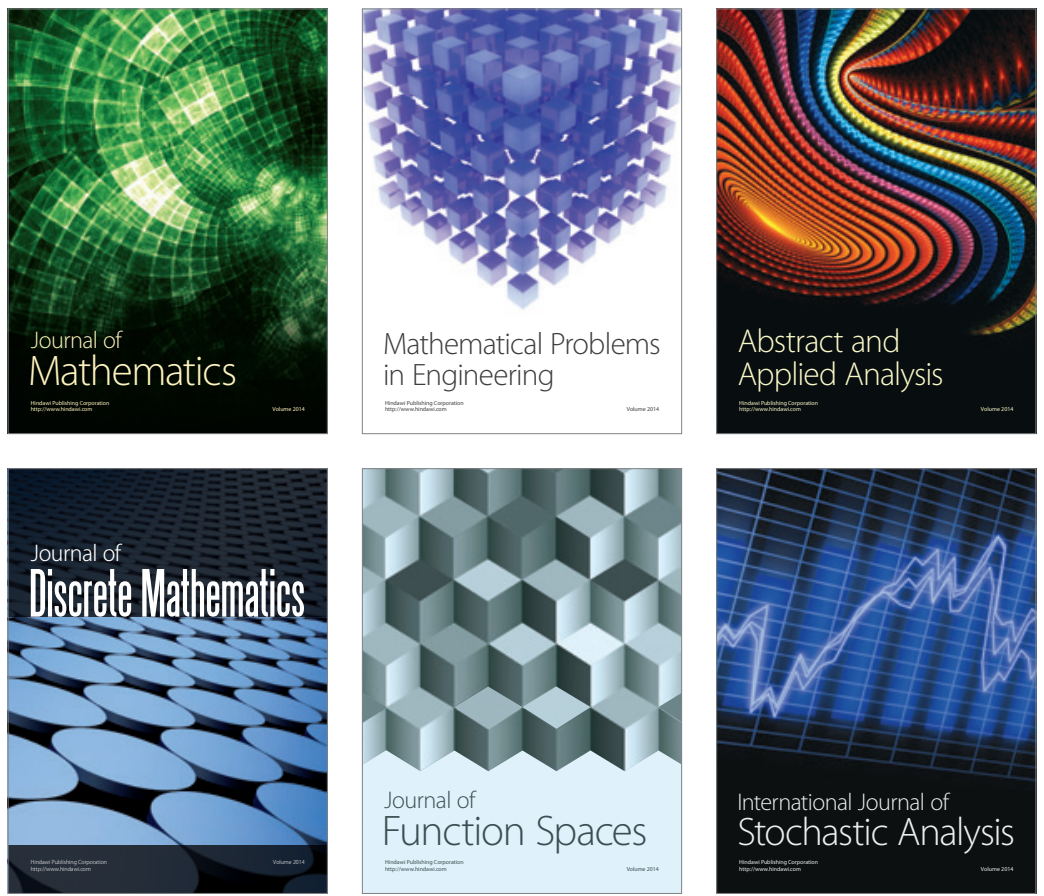

Journal of

Function Spaces

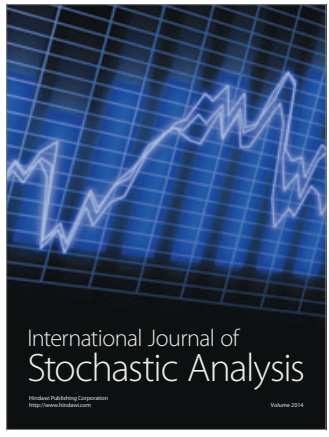

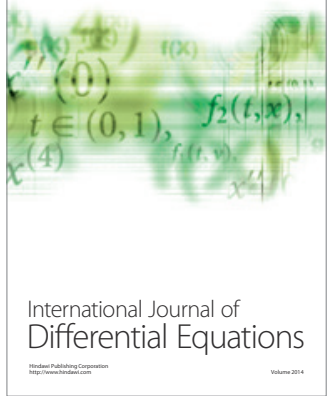
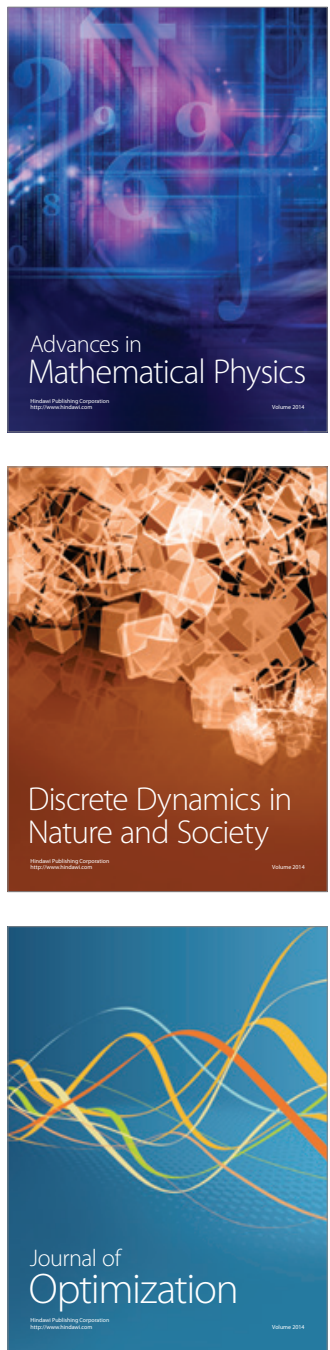